\title{
Semiotics and Chinese Tradition
}

\section{Towards a Semiotics of Chinese Characters}

\author{
Ting Liang (Ding Liang) \\ Professor, Department of Chinese Literature, National Taiwan University, \\ Taipei, Taiwan, China \\ lighting@ntu.edu.tw
}

\begin{abstract}
This article aims to describe the synchronic system of Chinese characters by investigating its cognitive trait of signs. Unlike alphabetic writing systems in which letters represent sounds, Chinese characters convey their meaning in part through images, which makes its synchronic system unique and requires a reconfiguration of the Saussurean framework of signifier and signified. I argue that the Chinese system is instead comprised of three components: pattern, meaning, and formation. Through a close investigation of the historical development of the Chinese character system and drawing on Peirce's classification of the sign, I propose a threefold adaptation of Saussure's semiology to Chinese that challenges the arbitrary relationship between signifier and signified, reconfigures the signifier from sound-image to visual character pattern, and proposes a morphological view that takes account of aesthetic principles in the generation and function of meaning.
\end{abstract}

\section{Keywords}

Chinese character - semiotics - synchronic system

In this article I analyse the signifying pattern of Chinese characters, which is neither arbitrary in the sense of Saussure nor a motivated relation in the 
sense of Peirce. It is well known that a linguistic system is the combination of two systems, namely the synchronic one and the diachronic one. The aim of this article is to analyse the synchronic system of Chinese characters which is unique because of its threefold composition (see Fig. 1): besides the signifier (which I will call 'pattern') and its meaning (signified), it also involves 'formation'. Formation includes principles of 'evaluation', 'imitation', and 'decoration', as described by Long Yuchun (1994). Scholars such as Heinrich Wolfflin ([1915] 1987) and Rudolf Arnheim (1974) have also acknowledged that 'aesthetic' principles should also be taken into consideration in the process of signifying, yet this principle is mentioned by neither Peirce nor Saussure.

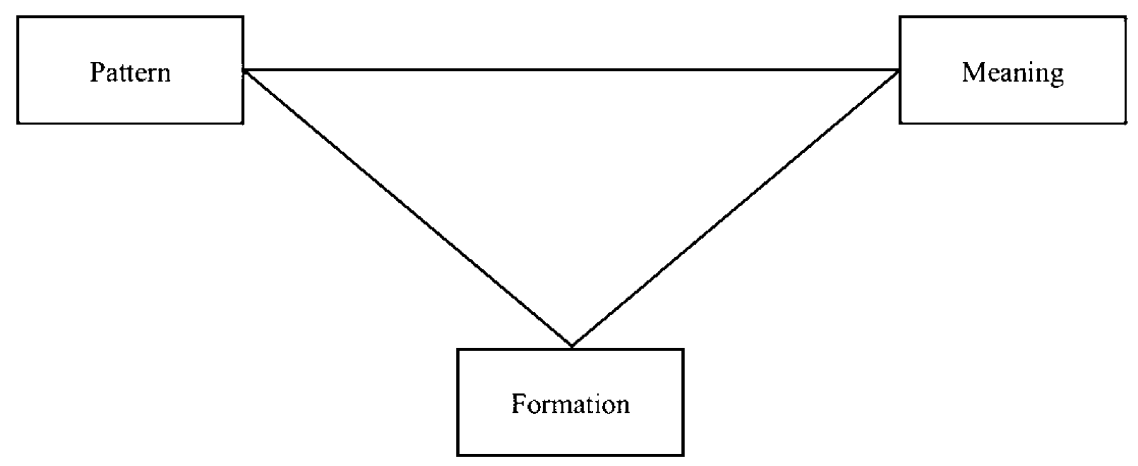

FIGURE 1 The synchronic system of Chinese characters (part 1)

A study of Chinese characters can thus reveal its importance more clearly, which is the aim of this article. In the following sections, I wiall also argue that with Chinese characters, there are two forms of meaning: 'container' and 'type'.

\section{Saussure's Semiology and Its Application to Chinese Characters}

It is well known that according to Saussure, a 'sign is a double entity, made up of the signifier and the signified' (2002: 135). This concise definition not only points out that language, as a sign, consists of two components, the signifier and the signified, but also highlights the arbitrariness of their combination. Once these two components coincide to create the sign, the meaning of the signified, which is imperceptible and elusive, is implied by the easy-toarticulate-and-identify signifier.

The pattern and meaning of Chinese characters must surely correspond to this definition. The meaning of a Chinese character is determined by identifying the perceptible differences in its pattern. For example, the number nine is written 九 in Chinese, while the character 丸 means 'round balls'. These two 
characters are similar in pattern with the exception of a stroke added in the second, which retains the articulation function of the signifier. We can refer to Saussure's signifier as the pattern of Chinese characters and the signified as the character meaning.

The arbitrariness of the combination of pattern and meaning is traceable in Chinese characters. Long Yuchun takes a basic standpoint in modern linguistics by combining the meaning embodied in the Xunzi:

Names have no intrinsic appropriateness. They are bound to something by agreement in order to name it. The agreement becomes fixed, the custom is established, and it is called 'appropriate'. If a name differs from the agreed name, it is then called 'inappropriate'.

XUNZI 1988: $682^{1}$

There is an arbitrary convention between the pattern and meaning of Chinese characters, differing only in the level of arbitrariness. For some characters, their meaning and patterns are related in a purely arbitrary way. The ancient way of writing the characters for the numbers four, five, six, seven, eight, nine, and ten are good examples (see Fig. 2). In particular, the pattern for the number four was changed to differentiate it from the number three, demonstrating the convention established in the number writing system (Long, 1994: 212).

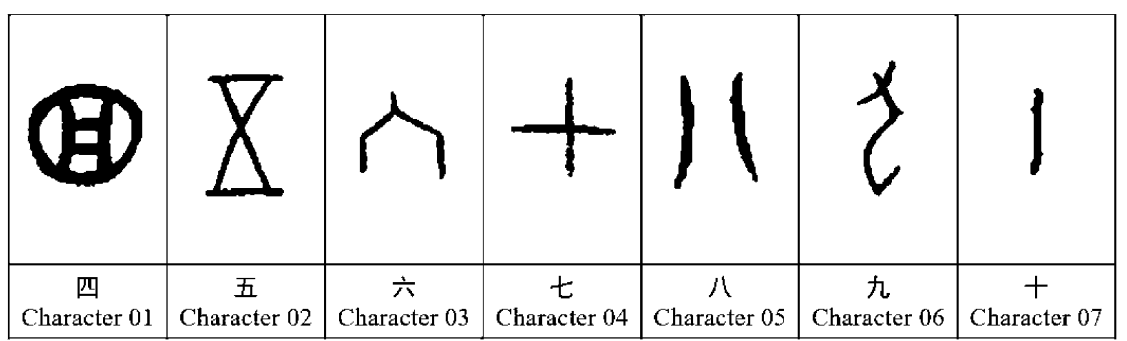

FIGURE 2 Characters of numbers

CHARACTERS 01-07, XU (1981: 548, 548, 548, 549, 31, 549, 84)

The second circumstance is that some strokes in the character pattern have no meaning other than to distinguish characters from one another, thus constituting arbitrariness. As is shown in the first two rows of Fig. 3, 田 (field) and 周 (complete, whole) originally had similar patterns, the second having one more dots. Similarly, 女 (female) and 母 (mother) had similar patterns. However, to distinguish them, the one with no dot was defined as 女 and the other as 母.

1 Unless otherwise stated, all translations are my own. 
The characters 月 (moon) and 夕 (evening), 星 (stars) and 晶 (originally means stars too and today means glittering) also fell into this category, sharing similar patterns in inscriptions on bones and tortoise shells during the Shang dynasty. They were then differentiated, with those with dots specified as 月 and 晶 (Long, 1994). The writing pattern of these characters is shown in Figure 3.

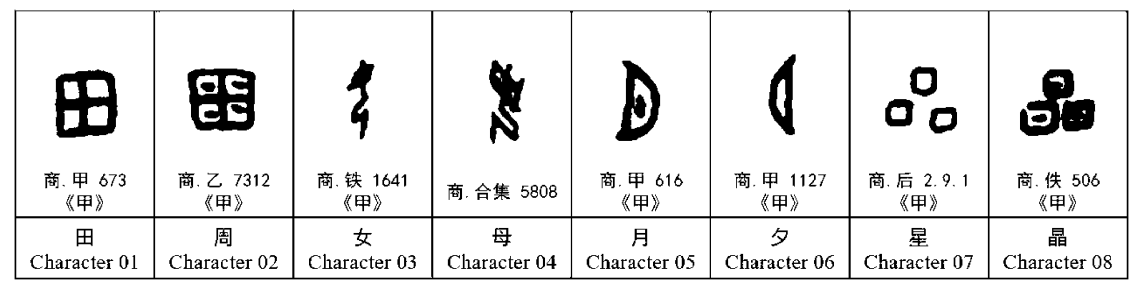

FIGURE 3 Arbitrarily designed characters

CHARACTERS 01, 02, 03, 04, 05, 06, 07, 08, JI (2010: 952, 95, 84, 887, 865, $571,562,561)$

Moreover, the four characters 史, 吏, 事, and 使 were the same, as were 桑, 丧, 咢, and 哥. Later generations distinguished these characters by adding or subtracting strokes, which can be considered a convention. The idea of adding or subtracting strokes broke the prejudices of philology of thousands of years, from the Han to Qing dynasties. Han Confucians believed that characters and strokes could be deconstructed, contributing to the compilation of the Shuowen Jiezi. As stated in The Family Instructions of Master Yan: Text Sources, 'if suspicion still remains, then you are in an utmost chaos and know little about the meaning of a single dot or stroke' (Yan, 199o: 458). Duan Yucai quotes this sentence in his preface to the Shuowen Jiezi, suggesting that traditional scholars believed that there is a reasonable association between the pattern and meaning of Chinese characters, while the convention indicates that no other association is required (Xu, 1981: 764). Long Yuchun considers this convention as one of the new six categories of Chinese characters (1994: 114-115).

Qiu Xigui (1993) takes this idea and renames it as 'mark'. He argues that many parts of characters which originally refer to meanings or sound gradually lost their original functions, and became 'marks' that are purely conventional (Qiu, 1993: 17).

Taking the character 并 as an example, it originally 'seemed like two people standing side by side', but 'when transformed into official script and standard style, the character turned into an indecomposable sign' (Qiu, 1993: 18). If the user of a language links pattern and meaning only according to his or her memory, the relationship between the signifier and the signified is arbitrary 
and generated by historical changes. Indeed, this circumstance is destined to happen since Saussure bases his study of language and signs on psychology, thus making the signifier and signified purely psychological phenomena.

Thus, if our ancestors had little knowledge of the origin of a specific character and used it from memory, then the pattern and meaning of this character were arbitrarily related. Saussure discusses alphabetic writing but never elaborates on this circumstance. Many Chinese characters are phonograms and ideograms, so the effective arbitrariness of the language is of greater importance. All Chinese characters are likely to operate according to this relationship. Due to its dependence on the cognitive status of language users, this relationship implies subjectivity, creating a fascinating and vital space for character development. As the binding force between the pattern and the meaning of characters disappeared during the cognitionacquiring process, and given their unchanged sound and pattern, Chinese characters needed to adapt to the needs of a period by assimilating new cultural connotations.

However, the application of Saussure's semiology to Chinese characters requires three adaptations already suggested in the preceding discussion. The first is to rectify the relationship between signifier and signified (Section 2 below). Compared with alphabetic writing, the relationship between these two in Chinese characters is not arbitrary. Many characters were coined using the 'motivated method', considered a trait of Chinese characters. As a result, there is more than one relation between signifier and signified in Chinese characters. Thus, another component, formation, is used in addition to the pattern and meaning of characters to contain both the arbitrary method and motivated method. This rectification affects the synchronic system of Chinese characters, which is at the centre of the discussion that follows.

The second adaptation is to rectify the description of the signifier by first adjusting the role or relationship between pattern and sound in the system (Sec. 3). The signifier refers to the pattern of Chinese characters, while Saussure considers it as a sound-image. As a result, the description shifts from auditory perception to visual perception. Second, it is rectified by entering into the connotation of the signifier of the pattern. As the character pattern is explicit and perceptible, a solid argumentation is easy to build, providing directions for future research on character meaning.

The third adaptation is to rectify the description of the signified, that is, the meaning (Sec. 4). When there are various probabilities in the relations between the signifier and the signified, the concept of the signified implied by the signifier through different relations can also vary. As a result, instead of a content-based view, a morphological view of the meaning of characters 
in the synchronic system is needed to support the entire system. Let us now take these three rectifications in turn.

2

\section{Formation: Associations of the Signifier and the Signified in Chinese Characters}

Apparently, the relationship between the pattern and meaning of Chinese characters is not purely arbitrary from the perspective of the six traditional categories of Chinese characters. According to Shuowen Jiezi: Postface (Xu, 1981: 755), composed by a Confucian of the Han dynasty, pictographs, one of the six categories of Chinese characters, such as 日 and 月 share similarities in their character pattern and the actual form of the object they represent. Similarly, picto-phonetic characters, like 江 and 河, have some associations in their sound and meaning. The list goes on. In other words, arbitrariness is not anecdotal. However, the Han Confucians' six-category theory is not an ideal theory of character formation for the modern academic field. Indeed, it was developed by self-proclaimed saints to unveil the will of heaven under the influence of Confucian ethical codes. In short, standard characters followed certain rules, on the basis of which the Han Confucians developed their theory to explain Chinese characters and interpret the classics. As recorded in Shuowen Jiezi: Postface:

The written language is the foundation of classical learning, the source of kingly government, the invaluable treasure our predecessors endowed us with to which our offspring resort for better understandings of the classics, thus 'once the fundamental rules were established, the great Tao would come into being accordingly'.

XU, 1981: 763

In other words, the six-category theory was advanced in reference to certain characters and based on character morphology rather than formation methods (Ding, 2002: 348-352). Nevertheless, character morphology is useful for defining the associations between the pattern and meaning of characters. Starting from the six-category theory, we can thus clarify these associations and discuss them from the perspective of semiotics after showing how the theory transformed into real associations between pattern and meaning.

In understanding the theory of six categories, Peirce's classification of signs is useful. A 'pictograph' which conveys its meaning through its character (such as the drawing of $\boldsymbol{C}$ to represent the sun 日 and to represent the moon 月) 
belongs to the category of 'icon'. Similarly, a 'pictophonetic character', which conveys its meaning by existing (such as the characters 江 and 河 whose left components are 'signific components' that refer to the meaning 'water' and their right semantic components 工 and 可are 'phonetic component' which indicate their pronunciations), belongs to the category of 'index'.

However, Long Yuchun argues that once the focus shifted to rules, the single component or different components of a character were no longer the basis of classification (1994: 220-221). Accordingly, some scholars consider characters like 取, 休, and 得 as 'associative compounds', meaning that because of their pictorial resemblance, their meaning can be classified as icon. Even an 'ideograph', one of the six categories that conveys an abstract idea through an iconic form, can be classified as icon, such as the directions 上 and 下 that express their meaning by an iconic indication above and below a line. In the case of associative compounds, a compound of two or more pictographic or ideographic characters suggesting the meaning of the word represented, such as the character 鲜, should be classified as index. Most Chinese characters are ideographs. If we consider 'mutually explanatory characters', ${ }^{2}$ which have 'the same etymological root' as the abstract form and the same extension of meaning, they belong to the index category along with 'phonetic loan characters', which are 'borrowed' to write other homophonous or near-homophonous morphemes. Indeed, according to the rules of association between pattern and meaning, the former function as signific components and the latter as phonetic components.

The 'symbol' in the Peircean sense can also be found in Chinese characters. According to $\mathrm{Xu}$ Shen, the character 武 ( $w u$, anything that is military) consists of 止 (zhi, to stop) and 戈 (ge, a weapon similar to the halberd) because the correct end of using military power is to stop the war (Xu, 1981: 632). Also, the character 信 ( $x$ in, truth, trust, or credit) is a combination of 人 (ren, human or people) and 言 (yan, words, speech), so that it means 'the words of a person should be trustworthy' (Xu, 1981: 92). Both are examples of symbols, for the formation of them are coherent with the general ethical belief in a specific historical background. It would be wrong to consider 止 and 戈 and 人 and 言 as two associated and independent signific components in the rules of index, or to turn 止戈 into 戈止 and 人言 into 言人. This illustrates the significant role played by rules in this process. In Shuowen, the numbers of similar examples are many (see Xu, 1981: 69, 86). When translating Buddhist scriptures and

2 Scholars offer various and differing explanations of this term. In this paper, these characters are defined as expressing abstract concepts through concrete situations, close to the extension of meaning proposed by the Qing Confucians. 
paternosters, medieval Chinese translators tended to combine two characters only to refer to special sounds. These combinations of characters can also be considered symbols in the Peircean sense (Long, 1994: 150).

As a result, another item, formation, should be added to the semiotic structure of Chinese characters, which is a combination of both the arbitrary method and the motivated method. It should be noted that formation is an independent item. Just as utensils are made by a combination of craft and $a$ particular craft that is applied to make many utensils, an existing Chinese character can match more than one formation method and a formation method can correspond to more than one type of character.

In practice, the formation method accurately reflects the variations and modifications of Chinese characters during the historical evolution process. For example, the three characters 凤 (鳳), 鸡 (雞), and 齿 (齒) in Figure 4 were originally pictorial drawings of the objects they represented. Later, the phonetic components 凡, 奚, and 止 were added to the three characters, making them the product of two categories combined, namely icon and index. The first two characters acquired the signific components 鸟 and 隹 only after the iconic parts 鳳 and 雞 had lost their distinctive features and assimilated 鸟. Then they belonged to the index category, with the signific components 鸟 and 隹 and the phonetic components 凡 and 奚. The last character used the same pattern after adding a phonetic component. Characters created by adding phonetic components and signific components defied enumeration, such as 暮, 曝, and 拔. Long Yuchun calls this phenomenon the 'transformation method' in the character formation rule of 'four creations and two transformations' (1994: 127-145). Even today, new characters are created using this method: for instance, 'watch' (表, biao) became 錶 and the Hakka dialect 'slat' (板, ban) became 粄.

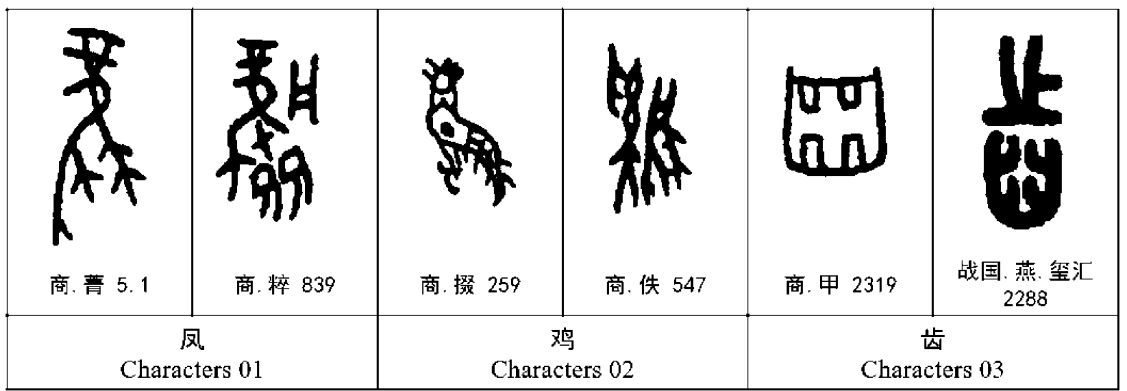

FIGURE 4 Characters of birds and teeth

CHARACTERS 01, 02, 03, JI (2010: 309, 289, 137) 
Moreover, 'formation independence' can help us differentiate and analyse the cognitive rule that similar patterns may come from heterogeneous patterns. For instance(Fig. 4), the aforementioned characters 凤 (鳳) and 鸡 (雞) with added phonetic components are different from the assimilated characters 凤 (鳳) and 鸡 (雞) with added signific components in terms of cognitive rules. Indeed, the latter have become abstract conceptions, while the former are on the cusp of it, still exhibiting pictorial conceptions.

In the same yein, the character 從, which was created by adding the signific components to the pictographic character 从 composed of two identical 人, still differs from the character 鲜 in terms of cognitive mode. The character 從 is on the cusp of abstract conception, while the character 鲜 has already adopted the signific components 鱼 and 羊. Similarly, comparing 鲜 with the characters 题 and 扁 leads to the same conclusion, although their character patterns are similar. Indeed, the latter two emphasize the rules of their cognitive mode, since their respective two abstract notions 是 and 少 were not arbitrary appositions. Unlike the apposition of 鱼 and 羊 forming 鲜, the first type contained a sequence order - as the reversed sequence 少 and 是 would make no sense. Taking into consideration the arbitrary method complicates things in this case. Indeed, the association between the pattern and the meaning of the character 凤 (鳳) can be treated as arbitrary, its users having little knowledge of its pattern evolution history and using it by pure recitation.

Indeed, language users can use a character when they only know a part of it. It is possible that one only knows the phonetic component 凡 in a character, and ignoring the part next to it. Following this reasoning, one can conclude that character formation depends on the pattern and the subjective knowledge of users. As such, formation independence highlights the role of cognition in character patterns, which leads us to recognize the cognitive schema of a period through the characters themselves, since signs are not just a record or reproduction but the incarnation of a particular era. Only by seeing through signs - the direct product of our mind - can we grasp the quintessence of cultures and observe the functioning of the human brain in certain cultures. In this way, we can become aware of the huge gap in cultural knowledge between the two mindsets, one reading 武 as 'charging forward with weapons in one's hand' and the other as 'using weapons and military forces to stop the war'. As formation is an independent term, Peirce's classification of signs into icon, index, and symbol needs to be adjusted and rectified theoretically. As a general rule, characters should not be classified by a single method or association; that is, in practice we cannot call a character an icon, index, or symbol, nor can 
we call it an arbitrary character. This single categorization can only be used in certain circumstances, generally for the sake of convenience.

Consequently, an association factor should come into play to account for the integration method of the signifier and the signified. For the sake of simplicity, formation is the name used to refer to this factor in this paper. It serves as the core in this system because of its relations with the signifier and the signified. Among these, the arbitrary association between pattern and meaning is conventional with few rules of interpretation, thus classified in the arbitrary method. Conversely, the other three associations between pattern and meaning, namely icon, index, and symbol, belong to the natural and cultural world inhabited by users with accessible rules of interpretation, thus classified in the motivated method. Therefore, a disparate semiotic system of Chinese characters consists of the three items: pattern, formation, and meaning (Fig. 1). With adjustments to the pattern and meaning of Chinese characters, a discussion of the signifier follows.

\section{Pattern: The Signifier in Chinese Characters}

From the perspective of the physiological function of the brain, the signifier in Chinese characters is the pattern and not the sound, which is an auxiliary tool. A sound was assigned to each character and listed as one of the three elements of traditional philology, the other two being pattern and meaning, due to the promotion of ancient Chinese phonology conducted by Qing Confucians. This is still the case for some philologists, such as Tang Lan, who pay little attention to ancient phonology (Tang, 2005: 61). However, from the perspective of Saussure's signifier and signified, their positions must be justified: the meaning corresponds to the signified, while the pattern and the sound correspond to the signifier. The pattern is the centrepiece of a character. It is thus essential for a character - even if the pattern and sound both exist physically and can be perceived and differentiated by our senses. In addition, objectively, the sound of a character depends on its pattern, particularly for non-alphabetic languages such as Chinese, in which a single character can have different sounds, thus highlighting the importance of patterns in the semiotics of this language.

Moreover, the visual effect of Chinese characters is cognitive. As Wang Shiyuan and Zeng Zhilang suggest, 'more visual memory is applied in identifying Chinese characters compared with alphabetic languages' (2002: 232-236). They argue that in a memory test, 'Chinese testees reflected better with the ones presented visually instead of auditorily while English tastes reported no such disparity' (ibid.). In reality, the pathways for recognizing Chinese characters 
and alphabetic languages are different in the brain, which can be observed in people who suffer from aphasia. Generally, lesions of the temporal lobe cortex severely affect the ability to read and write alphabetic languages, and damage to the occipital bone and parietal bone severely impairs the ability to read and write Chinese characters (ibid.). This indicates that the explanation that Chinese characters can generate direct associations between pattern and meaning because of their physiological basis, so a phonological record is not a prerequisite. Therefore, based on the cognitive and neurophysiological factors, the pattern of a character should be considered as its signifier in Chinese, while its sound is an auxiliary tool facilitating the association of character and language. ${ }^{3}$

In short, pattern-centred signifiers convert sound-based perception into visual perception. Saussure (2002: 112) recognizes the difference caused by this conversion:

Contrary to certain types of signs, such as visual signs by which complex objects can be represented from various dimensions, acoustic signs can only represent complex objects in one dimension. All elements of signs are placed in sequence.

Consequently, Chinese characters should not be confined to the 'acoustic' and 'chronological' features of the signifier proposed by Saussure. They not only assume the function of language records and its linear characteristics over time, but also demonstrate the features of two-dimensional visual signs by which complex objects can be represented from various dimensions, thus contributing to ancient Chinese bronze inscriptions, compound graphs, and characters of bronze inscriptions and oracle bone scripts - as well as to the quick reading habits of Chinese. Chinese characters place little importance on special expressions of alphabetical changes to prefixes, suffixes, and parts of speech over time.

The visual features of Chinese characters can be observed first in their pattern classification. Taking the example of an iconic character classified via the motivated method, its representation reveals 'vividness' through its depiction of an object or a circumstance. This representation can be generalized into three distinctive elements: namely, the form, direction, and position of the icon. The characters 鱼 and 车 evolved from their pictorial drawings of a fish and a carriage (Fig. 5). The meanings of 从 and 背 are differentiated by

3 The relationship between Chinese characters and language is an important and complex issue that deserves academic attention. 
the direction of the component $人$ in addition to their pictorial representation of a human being: the component $人$ has the same direction in 从 but the opposite direction in 背. Similarly, characters like 左, 右, 大, and 屰 distinguish their meaning by direction. As for the character 牢, the important thing is not the encircled animal - be it a cow (牛), a sheep (羊), or a horse (马) - but the position of the encircled objects. The same goes for the character 沈. It can be inferred that an icon is jointly influenced by form, direction, and position in two-dimensional visual perception.

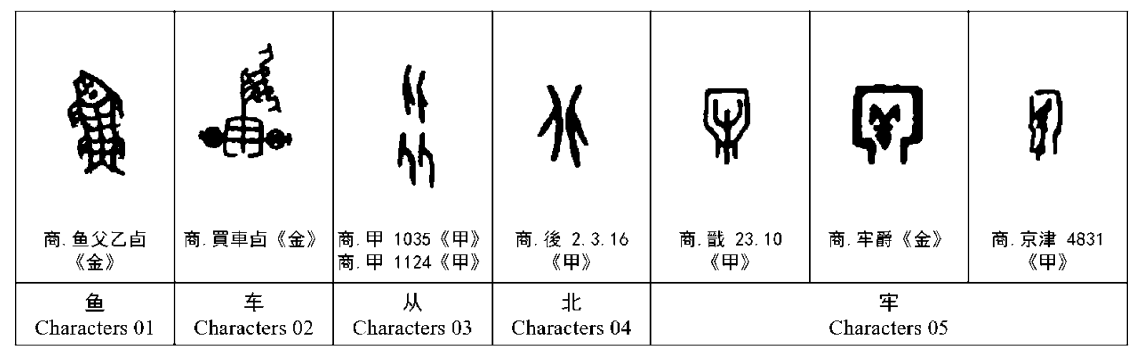

FIGURE 5 Characters with visual features

CHARACTERS 01, 02, 03, 04, 05 XU (1981: 853, 977, 670, 673, 92)

Using abstract reflection on phonetic components and signific components to make Chinese characters squarer, characters classified as indexes can combine each part according to 'humanism' and the characteristics of human visual perception, including top and bottom differentiation, left and right symmetry, and inner and outer distance. Discussing The Rites of Zhou:Baoshi, Jia Gongyan calls the characters created by this combination 'pictophonetic characters':

There are six categories of characters, among which the pictophonetic character is ubiquitous: such as characters like 江 and 河 with their pictorial part being the left, phonetic part being the right; characters like 鸠 and 鸽 with their two parts being the opposite; characters like 草 and 藻 with their pictorial part being the top, phonetic part being the bottom; characters like 婆 and 娑 with their two parts reversed; characters like 固 and 国 with their pictorial part being the outside, phonetic part being the inside; characters like 阙, 阍, 衡, and 衔 with their two parts being the contrary.

ZHENG \& JIA, 1989: 1575

Conversely, Wang Yun (1987:6) considers 阙 and 阓 as pictorial on the outside and phonetic on the inside, and 闻, 问, 誾, and 闽as pictorial on the inside and phonetic on the outside. The point is that Chinese characters can 
free variations in visual representations due to this visual pattern, with the exception of a few characters, such as 吟 and 含, 霖 and 棘, whose positions differ for the sake of meaning differentiation. In contrast, many characters are simply variants of the same character whose different positions do not contribute to meaning differentiation, such as 群 and 挐, 略 and 畧, 蚊 and 蚉, 峰 and 峯, 蟆 and 蟇, 裏 and 裡, 和 and 泺, 桌 and 棹, 雜 and 襍, 雜 and 襍, 松 and 昙 and 枀. This indicates that signifiers within Chinese characters can be the results of a series of 'dialogues' between sounds and visual forms. However, symbolic characters rely on the rules of meaning differentiation, which often refer to visually perceived sequences according to which character patterns are processed. This type of character has a feature of 'reproduction', such as the aforementioned 扁 (户 plus 册) and 匙 (是 plus 少).

Nevertheless, when considering the visual traits of Chinese characters from the standpoint of arbitrariness, the aesthetic function should be taken into account, in addition to meaning differentiation. With the aesthetic function as part of the list of considerations within the motivated method, based on meaning differentiation, there is still a margin of discretion in the arbitrary method. Taking 十 as an example, Long Yuchun argues that 'the character was originally written as $\mathbf{1}$, later as or evolved from dot to horizontal stoke, then to this existing one with a crossed horizontal and vertical stroke' (1994: 213) (Fig. 6). In fact, this ancient pattern variation phenomenon evolving from a slim to fat vertical stroke $>$ a fat vertical stroke to a fat stroke with a dot in the middle $>$ a dot in the middle to a short horizontal stroke $>$ a short horizontal stroke to a regular stroke intersecting with the vertical one, has become a rule guided by aesthetic principles. Yet, many philologists, such as Lin Suqing (1990: 32, 52), discuss this rule. Indeed, the character + in oracle bone inscriptions acquired its vertical stroke to balance its horizontal one. Although of aesthetic value, this transformation glossed over its original intention of formation. However, arbitrariness can still explain the association between pattern and meaning. This variation was ubiquitous, like the character 土, which was originally a pictorial drawing of a clod $\boldsymbol{\Delta}$ that was replaced by a vertical stroke, exactly like the transformation process of 十 (Fig. 7). The character 不, although retaining its middle vertical stroke, evolved into various patterns on Chu bamboo slips during the Warring States period under the influence of aesthetics (Fig. 8). Rules of little importance can be found in variations caused by aesthetics - such as 辛, which went from $\Psi_{\text {to }}$ by adding a horizontal at the top; or 余, which went from $\hat{\psi}$ to $\hat{x}$. Once the formation of a character moved from the motivated method to the arbitrary method, the pattern of the character was no longer confined to meaning disambiguation, but allowed to change freely for the sake of aesthetics. 


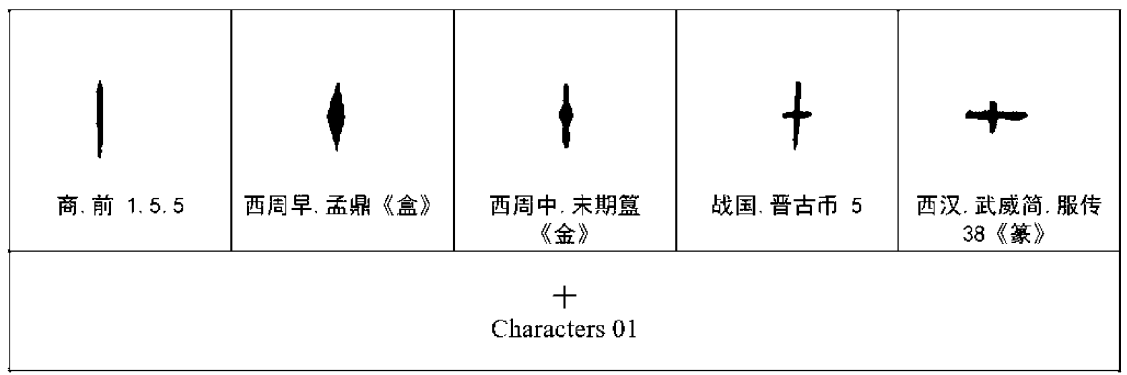

FIGURE 6 Characters of the number ten CHARACTERS 01, JI (2010: 153)

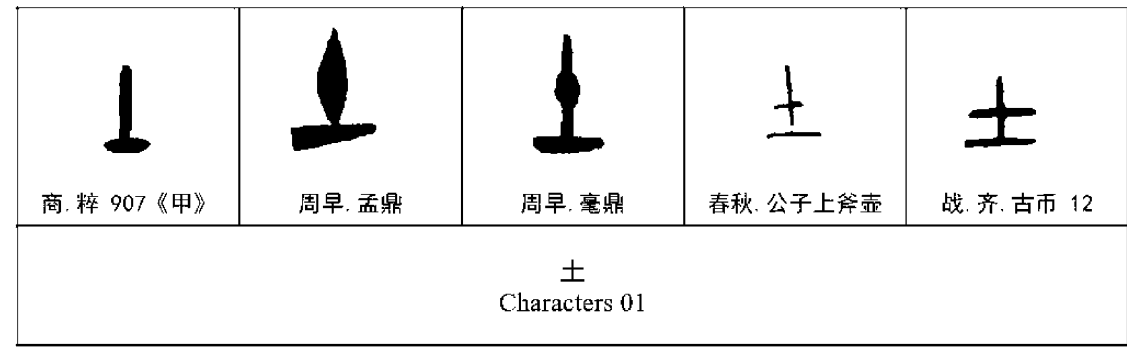

FIGURE 7 Characters of 'mud'

CHARACTERS 01, JI (2010: 944)

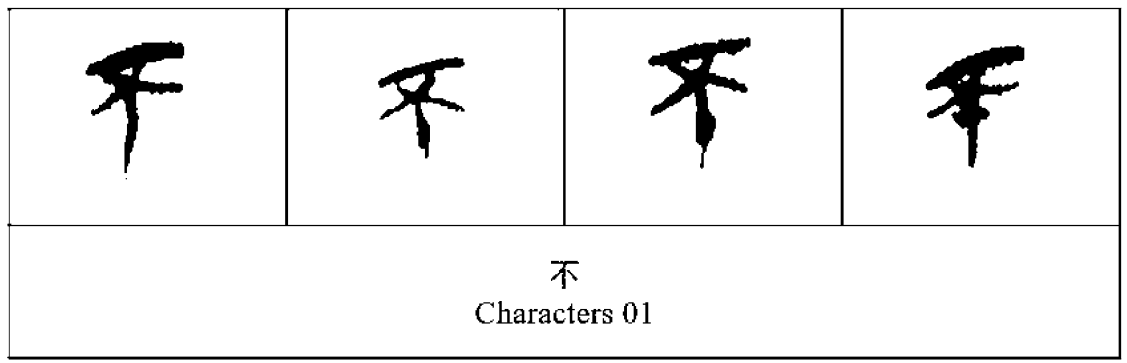

FIGURE 8 Characters of 'not'

CHARACTERS 01, JI (2010: 862)

In the semiotic system, these two factors interact. For instance, the initial pattern of $七$ was + , which was nearly identical to the post-variation character + . Therefore, for the sake of differentiation, ${ }^{+}$was later replaced by other patterns, such as $\mathbf{z}$, $\mathbf{t}$, and $\boldsymbol{t}$. Such interactions can be found in other characters; for example, 王 was 玉, and 工 was 壬.

Historically speaking, the major principle that underlies the aesthetic side of Chinese characters is the principle of 'assimilation'. Chinese philologists such as Long Yuchun (1994: 290-303) and Liu Zhao (2011: 95-105) highlight this 
principle of assimilation of character patterns. Figure 9 shows that the character 夫 (contained in characters 1-5) and 西 (character 6, which is contained in characters 6-9) are changed into different shapes when used as components, so that the whole character can look 'nicer' (Long, 1994: 290).

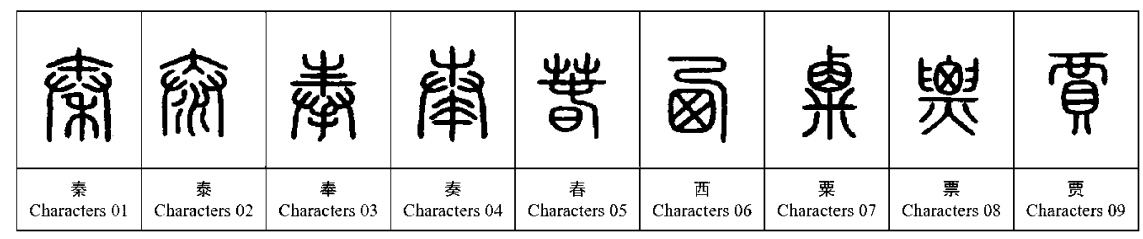

FIGURE 9 Different characters with similar parts

CHARACTERS 01-09, LONG (1994: 290)

Obviously, this phenomenon affects all Chinese character patterns, and this can be better understood with the help of Arnheim's (1974) philosophy of assimilation based on the Gestalt theory of visual perception. Arnheim highlights the basic structure of vision hidden in our cerebral cortex (Fig. 10), similar to the overlapping model of Jiugongge and Mizige used to learn Chinese calligraphy.
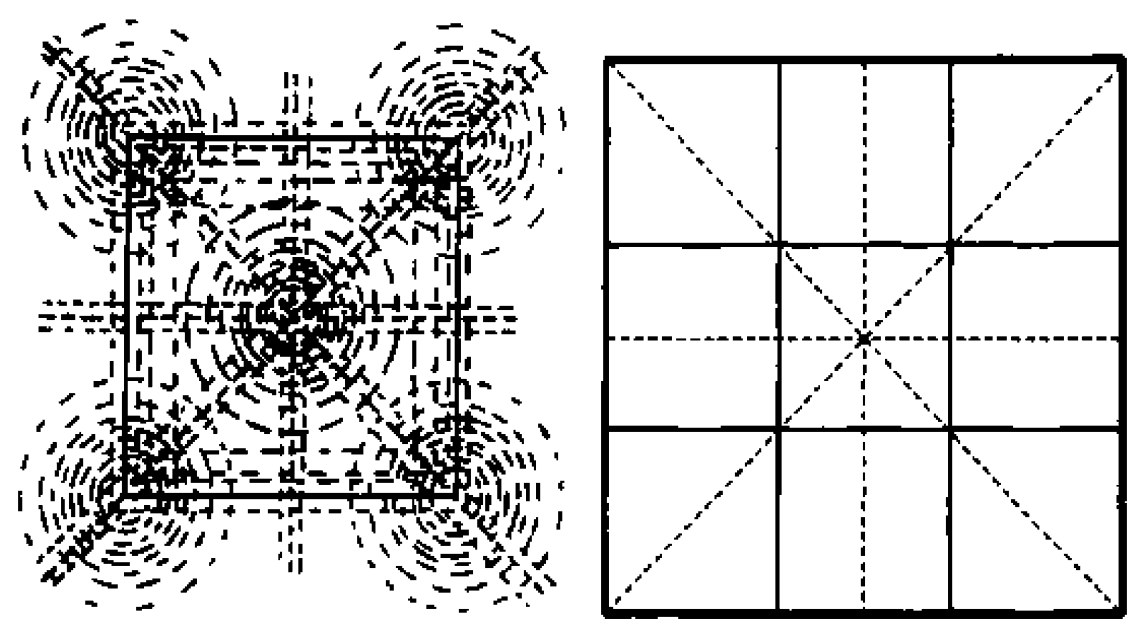

FIGURE 10 Basic structure of vision in the cerebral cortex ARNHEIM (1974: 13)

The balancing function of this structure differs from the mechanical imitation of the camera: the former actively participates in the visualization of objects, which is different from the optical replication of the latter. The features of the structure enable people to capture a panoramic perception of an object and leave out the minutia, making our vision a simple constitution. 
There are three different forms of the assimilation principle of visual structure of the Gestalt psychology: simplification, similarity, and grouping. Simplification consists in having a panoramic perception of a certain object through omission or a shortcut. For example, the modern forms of characters like 易, 车, 法, and 于 are the results of simplification (Fig. 11). The character 易 initially carried elements resembling a container and water, and was later simplified as the combination of 曰 and 勿. The pattern of 车 was simplified to a wheel and was similar to 亘 and 东. The character 法 abandoned its beastlike pattern for the simple combination of 水 and 去. While 于 was originally a pictorial drawing of the actual plant and was later simplified to the current pattern, similar to that of 干.

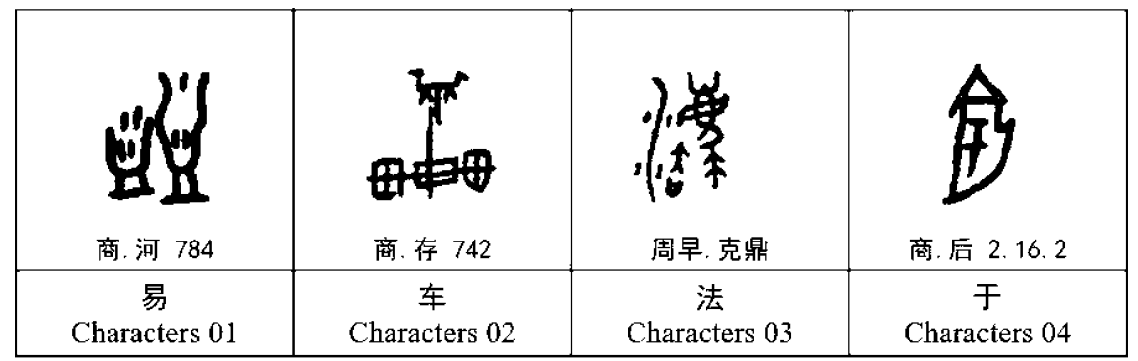

FIGURE 11 Characters before simplification CHARACTERS 01, 02, 03, 04, JI (2010:767, 977, 771, 404)

Simplification includes the phenomenon of similarity; that is, diverse character patterns were assimilated into similar ones, the integrated visual structure seeking similarities between various objects. The pattern variation of $⿴$, 甲, and 申, shown in Figure 12, is an example of similarity.

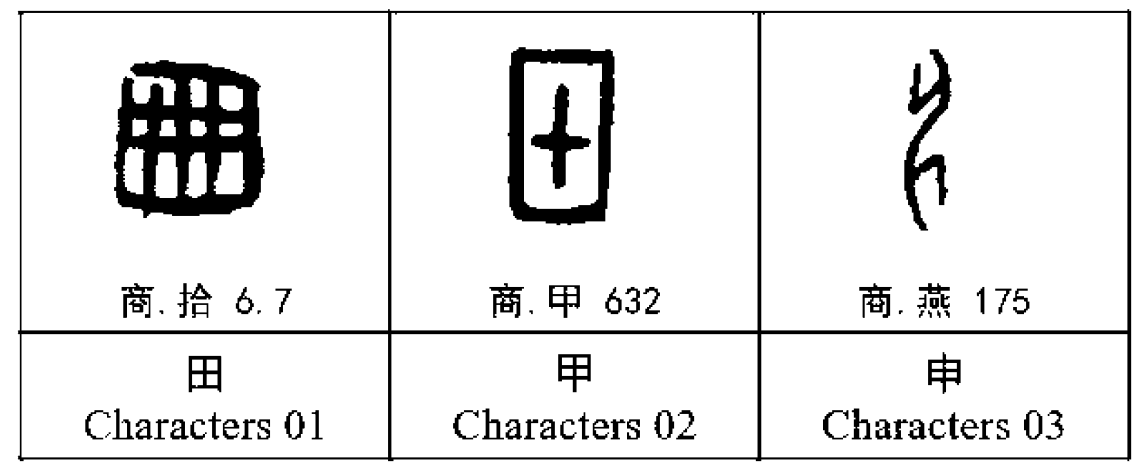

FIGURE 12 Examples of similarity

CHARACTERS 01, 02, 03, JI (2010: 952, 996, 1022) 
Finally, grouping consists in breaking down complex character patterns into a group of simplified patterns. The characters concerned generally depicted complex beings, such as 能 (熊), 龙, 鹿, and 燕, whose patterns were hardly recognizable based on their original pictorial depictions, but were easier to learn and master. Figure 13 shows the ancient patterns of 能, 龙, 鹿, and 燕.

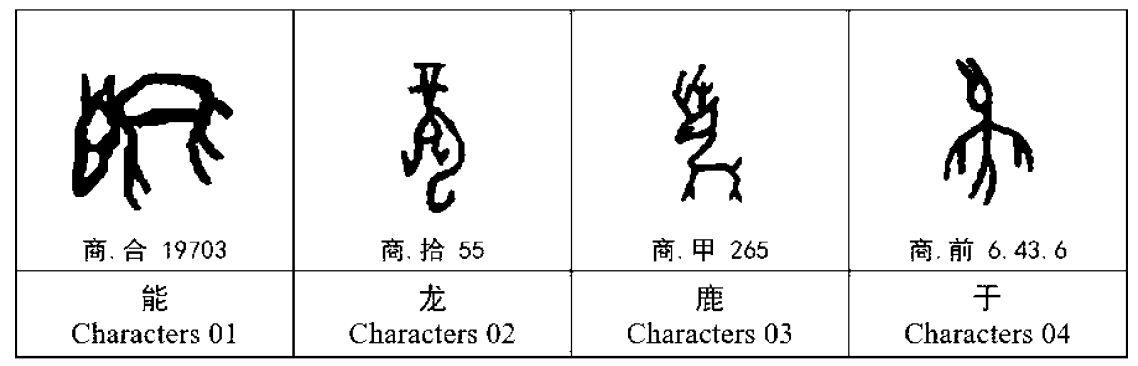

FIGURE 13 Characters of animals

CHARACTERS 01, 02, 03, 04, JI (2010: 781, 856, 772, 855)

In the case of 能, its original pattern was the complex pictorial drawing of a bear, which was then broken down into the simplified components $\angle$, 月, and $七$ through grouping. These three components are characters themselves. Moreover, the compotent $\Delta$ can be found in other characters, such as 私, 公, 松, and 么; 月 can be found in 肥, 滑, 肯, and 有; and 七 can be found in 比, 此, 牝, and 旨. As a result, the unique and complex pattern of 能 was assimilated into many other Chinese characters. The same goes for 龙, 鹿, and 燕.

Consequently, the signifier in Chinese characters has both the function of meaning differentiation and of aesthetic value. Long Yuchun (1994: 226) argues that the function of meaning and aesthetic values of Chinese characters are similar to the concepts of imitation and of 'decoration' coined by Heinrich Wolfflin ([1915] 1987:4O-41). I believe that Long's comparison between Chinese philology and Wolfflin's theory is in turn inspired by the theory of Wang Yun (1987: 118). Chinese characters can differentiate meanings by equating one pattern with another, so that meanings can be given in the consciousness of a reader. The aesthetic values of a character lie in the presentation of the pattern itself, which prioritizes the aesthetic value presented directly by visual perception. The characters formed by meaning differentiation originated from the appearance of various objects in the physical world, while those formed by aesthetic considerations stemmed from the neuromechanism of our cerebral cortex rather than the incidental or transient trends of a period. One of these two functions worked internally and the other externally. Together they shaped Chinese character patterns. In the future, the semiotic analysis of 
Chinese patterns may extend to the artistic styles of calligraphy or to the field of mentality and numerology in philology.

\section{Meaning: The Signified in Chinese Characters}

The signified in Chinese characters is their meaning. Meaning is an independent category in characters and is not determined by its content or by the general categories of content, such as astronomy, geography, minerals, plants, animals, or humanity. Instead, it must be determined by its traits and modes of operation in semiotics.

From the perspective of traits, the existence of meaning is neither the pure appendant of the physical world nor the product of ideology. Cassirer ([1925] 1990: 9-10) writes:

We must find in these forms themselves the measure and criterion for their truth and intrinsic meaning. Instead of taking them as mere copies of something else, we must see in each of these spiritual forms a spontaneous law of generation ... thus the special symbolic forms are not imitations, but organs of reality, since it is solely by their agency that anything real becomes an object for intellectual apprehension, and as such is made visible to us.

Signs are neither imitations of actual beings nor recordings of conceptions, thus meaning is independent of all things. In other words, the meaning of signs is not related to physical objects or to definite conceptions but to representations of intellectual orientations. Saussure considers this phenomenon as purely psychological, which implies that it is 'invisible' (2002: 13). This makes it more difficult to discuss. However, the meaning of signs is related to distinguishable patterns, thus the discussion of meaning must not be confined to the content of the signified but based on patterns and formations. In this way the modes of meaning are approached, and different modes of meaning are represented by different associations of the signifier and the signified. The discussion must be mode-centred rather than content-centred, as the content of meaning is irrelevant to semiotic structures, while the mode is the way for ideology to control ideas, which reveals the pattern of mental activity and is closely connected to the intrinsic structure of semiotics.

\subsection{Modes of Meaning: Container and Type}

Just as character formation is divided into arbitrary and justifiable, character meaning is divided into two corresponding modes: container and type. The 
container mode refers to the notion of being casual and abstract. If the relationship between the signifier and the signified of a particular sign is arbitrary, the signified is completely unrestrained, since nothing requires justification given its arbitrary association. Thus the signifier has no effect on the signified, be it guidance or containment. As such, the pattern is like a container with meaning placed inside, like wine, sand, or gas. As long as people agree to place a certain type of content in a particular container, which can be differentiated by shape, colour, or texture, we can tell its content instantly by its external features. Therefore, this mode distinguishes meaning based on discrepancies with other signs. Its structure is irrelevant to its content and creates a clear boundary to distinguish itself from other signifiers. As for its content, addition, omission, revision, and substitution are permissible whenever possible. Arnheim ([1969] 1987) refers to this as 'container connotations' and makes it the Western conventional form for grasping conceptions. The analysable form has clear boundaries and 'a set of attributes to determine a group of beings' (ibid., 267). Among the signs, the clear, definite, and logical mathematical sign is the most typical sign. For instance, $x$ can retain its casual and abstract features, since no actual object prevents its intention representation.

Merleau-Ponty uses Saussure's theories to argue that the container-based mode of meaning unleashes the content delivered, as meaning can be filled randomly and freely and 'each sign is like a blank form without any ink marks' ([1960] 2005: 108). As such, the user can replenish the content at will, which Merleau-Ponty calls 'my language' (ibid.). However, clear boundaries require unequivocal definitions. In Chinese, numbers like five, six, seven, and eight fall into this category: whether five apples, five trees, or five people, the number is irrefutably five. Similarly, from the perspective of arbitrariness, in which patterns are unjustifiable and memory serves to bind the signifier and the signified, the meaning of a character is inclined to be casual and abstract. Indeed, as recorded in Chuang Tzu: On the Equality of Things, 'a path is formed because we walk on it; a thing has a name because we call it so' (Zhuang, 1991: 69). Certainly, if meaning is implied by highly abstract expressions, we try to grasp it using the container mode, as it accommodates abstract connotations that are hard to describe and independent of all that is real.

The type mode refers to conceptions that are concrete and restricted, corresponding to the motivated method. First proposed by Arnheim, this mode means to perceive the structure of a concrete experience through intuition and to represent the essence of a certain situation or object by its complete picture. Thus a type notion is established to represent 'the structural essence of such a kind of entity' (Arnheim, [1969] 1987: 267). The core of meaning becomes clear and its extensions to the outside world are guaranteed, but the degree and status of these extensions depend on the circumstances, obscuring the 
boundaries between conceptions. In the case of Chinese characters, it refers to the traits of the signified, which evolve with the associations between the signifier and the ways of signifying, such as the ancient scripts 月 and 夕, both of which bear the pictorial drawing of the moon, with the former being moonrelated and the latter time-related. Linguistically, these two are like comparing apples and oranges with their own distinct attributes. Yet they share similarities in their pattern. As the moon-shaped conception is a product of visual perception and a mixture of substance and time, clear boundaries cannot be defined. But it has a core meaning that refers to the real moon in the dark night, which will never turn into sun or daytime, regardless of changes in the meaning of the word. In this mode of meaning, the moon will never become its opposite. The same goes for 帚 and 妇, 示 and 祇 (Fig .14): the two pairs differ in their sound, but the first pair adopted the drawing of a broom and the second pair the drawing of a sacrifice. In other words, the real being reflected by the pattern serves as the core meaning, which then extends its boundaries and diversifies its meanings. However, reverse transformations are never possible.

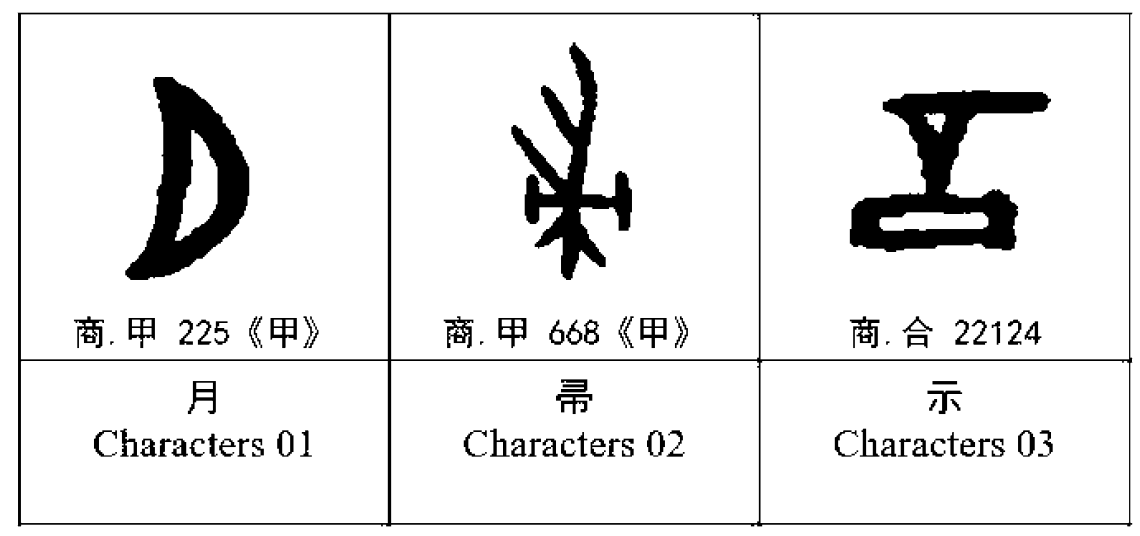

FIGURE 14 Examples of type mode

CHARACTERS 01, 02, 03, JI (2010: 565, 643, 45)

\subsection{Entitative Connotation, Associative Connotation, and Cultural Connotation}

Similar to Peirce's classification, signs that are 'types' within Arnheim's ([1969] 1987: 267) theory can also be classified into three forms: entitative, associative, and cultural.

An entitative sign is a spiritual reflection of real beings. Life experiences are stored in our memory and converted into a unified perception in specific situations. The characters 月, 帚, and 示 are good examples. Obviously, we build our relation to the real world with the help of such signs. It should be 
noted that the concrete reflection of an entity is a mere representation of signs in semiotic terms and that its actual incarnation may not exist in the outside world. For example, 鬼 and 龙 in bone inscriptions indicate that people of that time believed in such things as ghosts and dragons, which does not mean that these beings exist in the real world. The character of 人 (human) can be used in 仁 (benevolence); 凤 in 风; 熊 in 能; 蛇 in 它; 桑 in 丧; and 羊 in 祥 ( $\mathrm{Tu}, 2006$ : 274). In addition, these signs have been subject to influences from the historical world since its inception. The related entitative connotations of 羊 will be explained in detail by a group of 羊-based characters.

羊, 姜, 㒸, 美, 岳, 义(羲), 祥, 详, 善, 膳, 恙, 差, and 㦈 are a group of 羊-based characters. Apparently, 羊 is the pictorial drawing of a sheep's head as the representation of the sheep itself and its entitative connotation was influenced by the sheep-worshipping custom of the ancient Qiang ethnic group, contributing to the formation of a series of 羊-based characters (Fig. 15).

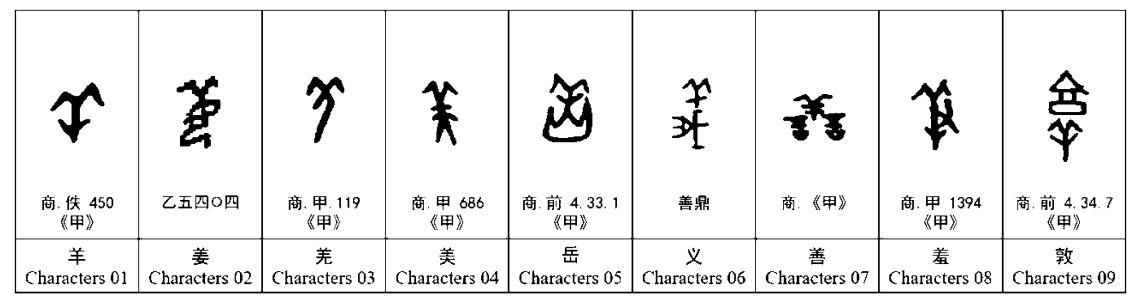

FIGURE 15 Characters related with the character of 'sheep' CHARACTERS 01, 03, 04, 05, 07, 08, 09, JI (2010: 302, 304, 303, 749, 1016, 245); CHARACTERS 02, 06, 07, XU (1981: 432, 483, 96)

How are the characters 姜, 㒸, 美, 岳, 义, 善, 差, and 敦 connected to 羊? Besides interpretations from traditional philology, it is also useful to take Freud's (2006: 157) psychological study of totem into consideration:

Among the Australians the system of Totemism takes the place of all religious and social institutions. Australian tribes are divided into smaller septs or clans, each taking the name of its totem. Now what is a totem? As a rule it is an animal, either edible or harmless, or dangerous and feared; more rarely the totem is a plant or a force of nature (rain, water), which stands in a peculiar relation to the whole clan. The totem is first of all the tribal ancestor of the clan, as well as its tutelary spirit and protector; it sends oracles and, though otherwise dangerous, the totem knows and spares its children. The members of a totem are therefore under a sacred obligation not to kill (destroy) their totem, to abstain from eating its meat or from any other enjoyment of it ... from time to time festivals are held 
at which the members of a totem represent or imitate, in ceremonial dances, the movements and characteristics of their totems ... . A member of a clan seeks to emphasize his relationship to the totem in various significant ways; he imitates an exterior similarity by dressing himself in the skins of the totem animal, by having the picture of it tattooed upon himself, and in other ways.

The sheep is the worshipped totem and the deemed ancestor of the ancient Qiang minority. They named their group 㒸 and adopted 姜 as their last name. As the totem of the Qiang people, the sheep can send oracles and spare good from evil, thus proclaiming their righteousness (义), from which another character which means 'discussing' (议) was formed. The totem also represents benevolence (善) because it provides assistance to its people. Moreover, the sheep excels at mountain climbing and those who reach the summit will be revered as deities, i.e. high mountains (岳). The sheep knows and spares its children, although it is dangerous for other clans, which can be seen in 祥, which represents auspices and omens for disasters, and in 详 indicating the sharp eyes of the sheep to identify its people and distinguish right from wrong. Therefore, the Qiang people imitate an external resemblance to the totem. In return, 㒸 probably comes from the image of its people wearing its skin or its head. Some dance movements during ritual ceremonies are imitations of the sheep totem, which can be seen in the character 美 (beauty). The Qiang people should refrain from eating its meat; those who do, violate taboos, as this act is disgraceful or shameful (差) in Chinese.

However, the slaughter of sheep is allowed at grand ceremonies or festivals as a gesture of worship to the sheep deity, thus creating the character 敦 for culling and 炖 for stew. After the ceremony, the meat of the sacrificed sheep is divided and shared among its people, which is treasured (嵄), evolved from 差. Those who are lucky enough to share its meat receive the blessings of the deity, thus having a meal is 膳 in Chinese. Those who help during the worship ceremony can get their share of meat and are called 膳夫 (people in charge of dining). Those who fail to receive a share can only envy (羡) others or catch a disease (恙) in worse situations (Huang, 1955). In conclusion, the entitative connotations of this group of characters were constituted with the entity 羊 at their core and the independent and unique circumstances of totem-worship practices in ancient times as the contributing force.

Non-entitative meanings, such as disposition, affection, and understanding, can be reflected by abstract connotations, which are based on pictographic characters in Chinese and supplemented by associations with sound or meaning. Associations enable meaning to be free from concrete connotations, to some 
extent, and allow real-life experiences to become the bridge to the abstract category of 类. Lakoff (1994: 79-108) uses idealized cognitive models as revealed by prototypes in his book Women, Fire and Dangerous Things, in which he points out that this connotation functions as a category that highlights the core and blurs the boundary. The relations between the components and their characters recorded in Shuowen Jiezi reveal the mode of meaning of abstract connotations. Taking the example of the header 心 in the tenth volume, 心 is a pictorial drawing of a heart. However, its abstract connotation is not constrained to the organ itself but serves as reflections of certain external objects with perceivable temperament, subjective attitude, and acceptance of changes. The characters 息, 情, 性, 志, 意, 恉, 息, 应, 慎, 忠, and 壳 are all based on this header, and as such have meanings related to and centred on the character 心 (Xu, 1981: 501-515). Moreover, according to the traits of meaning, different abstract meanings are mutually substitutable in character application, such as 言 and 心, and 9 and 莫. However, different characters in one language work in parallel. Shen Jianshi (1935: 785), a prestigious scholar of exegesis, introduces and extends the practical use of this connotation, citing Tang Song shi liao biji cong kan by Zhang Shinan:

As Shuowen blazed a trail by its category of graphs and components, Yupian followed the path as a sequel. However, both overlooked the fact that components to the right of the character also demonstrated rules. As [the] character 戈 means shallow and tiny, so waters that can wade through is described as 浅; those who have deformity are called 残; goods that are cheap are described as 贱; woods that are light and thin are called 栈. [The] [c]haracter 青 implies sagaciousness, thus unsheltered places under the sunlight are known as 晴; waters that are muddy churning are deemed as 清; things that can be clearly seen are described as 睛; rice that removes the crust is called 精. This type of character can be inferred, and I just list two examples here for you to have a brief glimpse.

In this case, meanings are entailed by categories, enabling the characters to represent pure abstract connotations. Even concrete representations, such as shallow water, are expressed by abstract categories. Thus, the character 情 with the greatest abstractness can use this method by adopting two signific components 心 and 青 to convey in alliance its associative meaning. 青 probably indicates blue, which is extracted from bluegrass for its fine, pure, and better colouring. The upper part of 青 is related to 草 and the lower part to 丹 to indicate that 青 is extracted from bluegrass. 青 also represents the most valuable and refined ingredient in bluegrass and can be used to indicate abstract 
connotations, just as 情 refers to the inner sincerity of one's mind and 请 to the authenticity of one's words.

The signific component of 青 recalls the meaning of 情. The phonetic component can also refer to its associated meaning, just as 吾 alludes to 明 and 逆. Moreover, as is shown in Figure 16, the characters related to 明 are 悟, 㝥, or 唔, and the characters related to 逆 are 啎, 圄, 敳, 语, 嶈, or 龉 (Shen, 1935: 817). Shen recompiles Guangyun in accordance with the pictophonetic rules, creating a systematic compilation of character meanings. Because the associations between the signifier and the signified have widened their gap, meanings have more freedom and similar patterns can result in different associations. For instance, 红 may represent different meanings, such as colour and needlework.

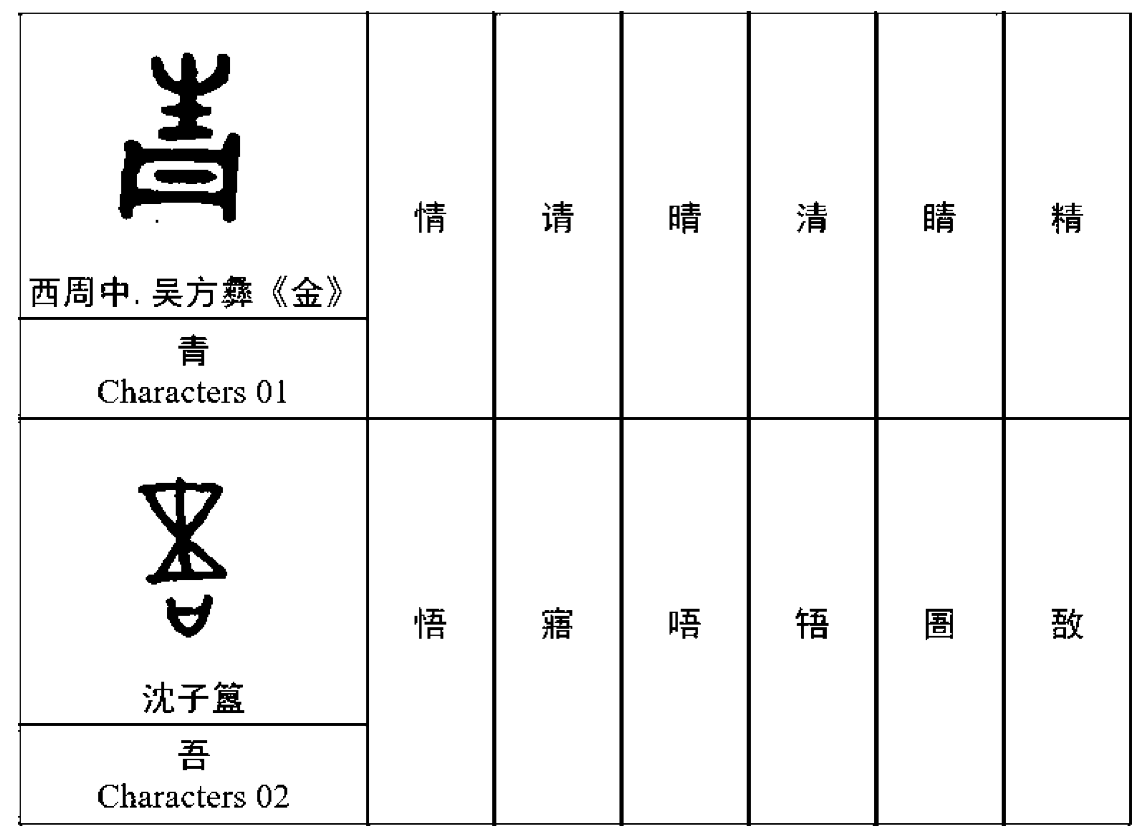

FIGURE 16 Characters based on association

CHARACTERS 01, JI (2010: 437); CHARACTERS 02, XU (1981: 40)

A cultural connotation adds another consideration to an abstract connotation and creates associations between different characters in accordance with the culturally approved mode. Apparently, the connections approved by this mode have strong cultural features, such as the mathematical symbols $\sum$ and $\log$. In this way, meanings are generated in one system, enhancing their systematic argument. For example, the sounds of the aforementioned characters coined during the translation process of Buddhist scriptures and paternosters 
in Chinese middle ancient times by combining two characters - like 名 and 夜, 亭 and 夜, 卑 and 也, and 宁 and 也 - are determined according to the Fan Qie 反切 system ${ }^{4}$ based on the theory of transliteration at that time. Moreover, these sounds are considered as spells or curses that have super-natural power in the Buddhist religion. Similarly, Scriptures of the Great Peace, which emerged during the Eastern Han dynasty, can be viewed as a system founded by the pattern of 'Compound Characters' under the influence of Taoism (Fig. 17).
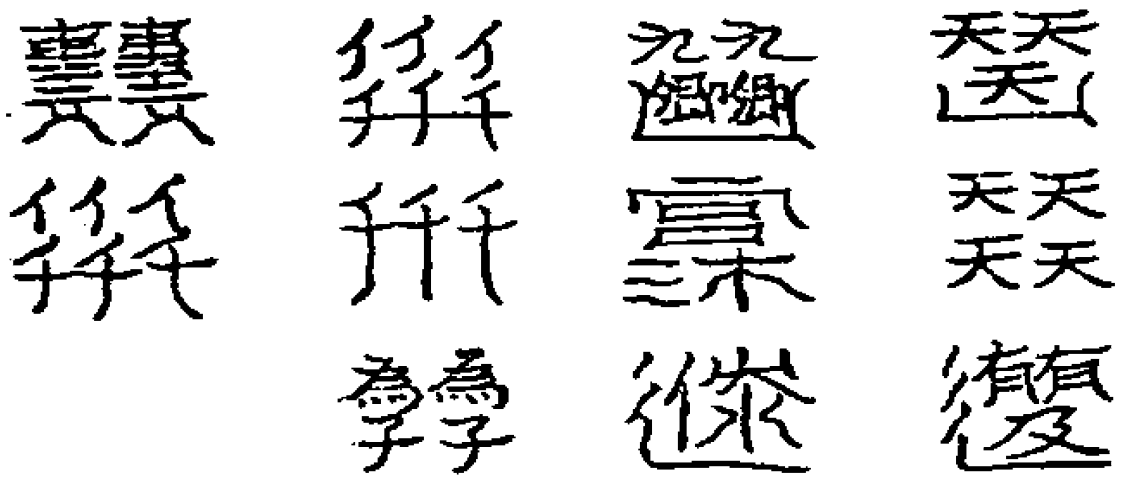

FIGURE 17 'Compound characters' BASED ON WANG (1960:483)

Although it is difficult to retrace the exact meanings encoded in compound characters, Taoist incantations and magic symbols were derived from these characters. Surely, the most common connection method was based on characters and obtained by recombination, slight modification, or reinterpretation of the components. The compound method prevailed over naturalism during the Wei, Jin, and Northern and Southern dynasties, as stated in Yan Zhitui's The Family Instructions of Master Yan: Miscellaneous Arts:

The Northern dynasty has gone through [the] turmoil of long-lasting wars, [and] now its characters and writings are unsightly and repulsive to the eyes; worse than that, they fashioned new characters without authorization, resulting in uncouth and unwieldy style which even beat the regions south of the Yangtze river. So much so they combined 百 and 念 to substitute the character 忧; 言 and 反 to substitute the character 变; 不 and 用 to substitute the character 罢; 更 and 生 to substitute the

4 Fan Qie is the traditional Chinese alphabetic system within which characters are used as letters to refer to voices. 
character 苏; 先 and 人 to substitute the character 老. Such alterations were ubiquitous.

YAN, 1990: 514

Yan criticizes the practice of coining characters, but those characters he mentioned were the product of assembly language, which became the epitome of culture at his time. The character formed by 百 (hundred) and 念 (thoughts) slightly modified the pattern of 忧 (worry) but reflected the Buddhist thought that worry comes from too much thinking. Similarly, the character formed by 言 (words) and 反 slightly altered the pattern of 变. Their combined pattern described academic debates among celebrated scholars with no official position. Some characters are still in use today. For example, the modern character of 'body' (体) is formed by the combination of the part $\uparrow$ which is changed from the character 人 (human) and the part from 本 (root), so that the whole character is understood as 'the body, which is the root or the most important part of a human being'.

Thus, the meaning of Chinese characters is multifaceted. The first basic mode is container, that which contains its liquids. It has clear boundaries and content that is not confined to the form of the container but characterized by openness, enabling free and abstract expressions to cope with new changes in the world. The second is type, which is based on real entities in the world, with a stable core meaning and whose boundaries are unclear and versatile, producing constant existences that are difficult to differentiate yet not easy to change. This second mode can be further classified into entitative, associative, and cultural connotations, with the first adding a cognitive layer to form the second: that is, an associative connotation is formed by adding an associative cognition to an entitative connotation. A cultural connotation is formed by adding a cultural cognition to an abstract connotation, in accordance with Peirce's discussion (Tu, 2006: 274). The container mode frees the meaning of signs, while the type mode stabilizes it, the two working together to develop and transform the meaning of characters, so as to satisfy the requirements of the Chinese writing system.

This paper has analysed in sections the essential constituents of the synchronic system. But the system is best known for its overall functioning, which contains its structure, the interactions between each constituent, its operating attitude, the influence of culture, and the transfer to the outside. 
The synchronic semiotic structure of Chinese characters is based on three elements: pattern, meaning, and formation. Each element can be further divided into two influencing factors: for pattern, these are meaning differentiation and aesthetic consideration; for formation, the arbitrary method and the motivated method; and for meaning, container and type. These three elements can be treated as being independent from each other, so that the semiotic structure of Chinese characters can be described in Fig. 18:

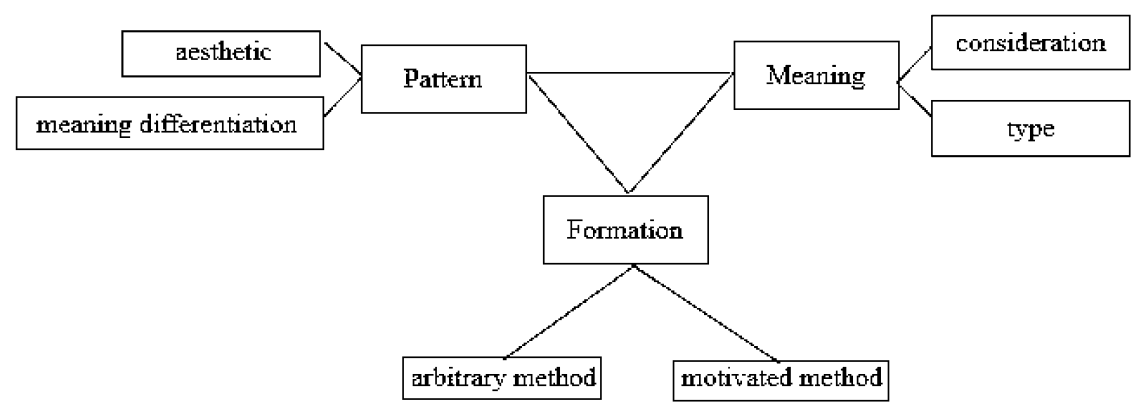

FIGURE 18 The semiotic system of Chinese characters (Part 2)

Each constituent of the structure interacts closely with the others, since signs are synchronic and inseparable. Therefore, changing one part will lead to chain reactions throughout the system. For example, modifying the pattern of a certain character will change its association with meaning, facilitating formation changes, and a new formation contributes to a new interpretation, thus generating a new meaning. Therefore, pattern, formation, and meaning should be analysed within this system rather than in isolation. Consequently, this semiotic structure enables Chinese characters to forge a cultural integration transcending time and space. As one of the most influential sinologists, Karlgren (1985:14-15) explains this fact as an outsider of Chinese culture:

Chinese characters are independent ideographs instead of phonetic graphs, so whatever changes to their sound, however mixed up of their tone, their writings will always be distinguishable .... Chinese people have a taste in imitating ancient styles, contributing to the formation of a polished and concise writing style .... Thus, a line is drawn between 'classical Chinese' and 'vernacular Chinese' .... Diachronically speaking, classical Chinese learning will enable us to get an understanding about the official dispatches from some thousands years ago regardless of their change in sound and tone .... Synchronically speaking, each one of us can interpret these writings correspondences in our own way; as such 
classical Chinese may have lost their value as a spoken instrument, but more as a written Esperanto for accessing official servants .... Classical Chinese character is the foundation of the whole Chinese culture, and a bond that ties our people together to unite as one big country. Such may be the power of classical Chinese!

Karlgren notes the cultural integration efforts of classical Chinese and compares it with alphabetic writings with no regard for the operational attitude of the semiotic structure. He concludes that the two systems differ from each other because alphabetic writings are a language recording tool. Therefore, Chinese characters can be separated from spoken languages to form 'a kind of written Esperanto' that appeals to the eye, so as to forge a classical Chinese writing system that transcends time and space. He compares the European division with the long history and integration of China, due, according to him, to Chinese characters. Indeed, the idea that language does not confine characters can enable Chinese to create a suitable semiotic system beyond language for people or facilitate its application among people around the world in transnational networks. As such, Chinese is in a position to become a new Esperanto. In fact, its semiotic system has unintentionally demonstrated its influence in various cultures.

Filled with rich connotations, the synchronic system of Chinese characters can use its flexibly to form its own semiotic structure or affect those of another culture. This transformation can be roughly divided into three categories: pattern-meaning, pattern-sound, and pure-pattern. The first is similar to the indicative function of signific components in the motivated method. Taking the example of Chinese applications in the cultural circle of East Asia, those adopted by Japanese people localize sounds with nearly identical patterns but different meanings, contributing to the poly-sound of a particular pattern, which is a distinct proof of the constraint-free characteristic of Chinese characters. The second, pattern-sound transformation, has the indicative function of phonetic components and can convert them into the phonetic system of alphabetic writings in the arbitrary method. For instance, hiragana and katakana in Japanese are phonographs borrowed from Chinese, though characters within these two systems actually function as letters in an alphabetic system. In fact, from the perspective of the purely semiotic system, the evolution of Chinese character styles, namely lesser seal, official script, regular style, running hand, and grass writing, can be considered as a transformation over time. In the same vein, the transition from inscriptions on the ancient tally, ancient imperial seal script, inscriptions on ancient bamboo weapons, bird-worm seal script used on flags, characters for Taoist incantations, ancient imperial edict script, and even traditional and simplified Chinese characters can be seen as a transformation in character application. 
Based on the overall functioning of the synchronic system, we can see the versatility of Chinese characters as intertwined and independent, constant and flexible, ensuring their long circulation and generating many enriching chapters, thereby making Chinese the deepest semiotic system of any human civilization.

At the beginning of this article, I give a simple diagram (Fig. 1) to portray the synchronic structure of Chinese characters. Now that I have studied each of the three parts in turn, this structure can be described in more detail (Fig. 19).

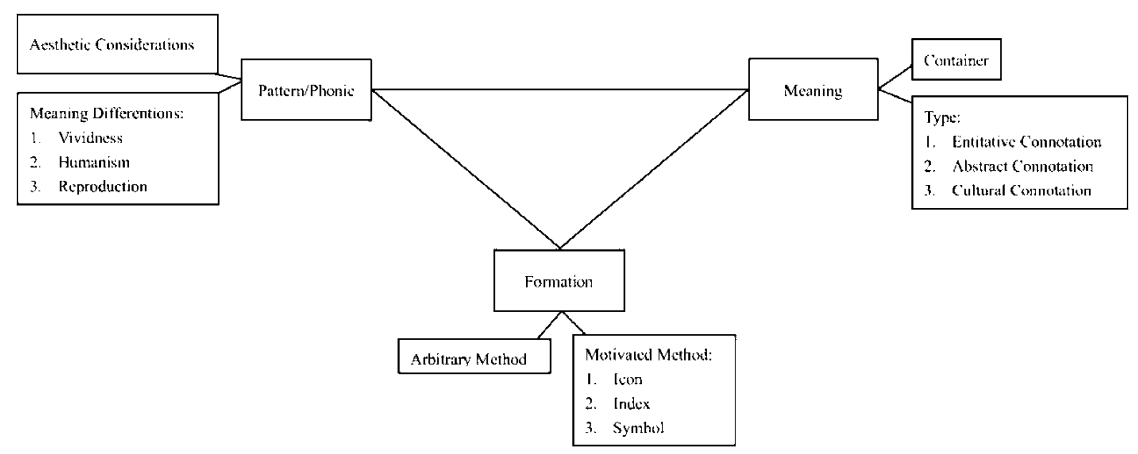

FIGURE 19 The synchronic system of Chinese characters detailed version

The independent and close interactions of each element in this system make it an all-embracing system with many different evolutions over time. Historically, this system helps maintain social order and adapt to changes over time to nurture a deep and far-reaching culture. By offering a structural description of this system, I believe that this article can prepare a formal ground for future studies on the essence of the culture itself.

\section{References}

Arnheim, Rudolf (1974) Art and Visual Perception. Berkeley: University of California Press.

Arnheim, Rudolf ([1969] 1987) Visual Thinking (视觉思维, Shijue Siwei). Trans. Teng Shouyao. Beijing: Guangming Ribao Chubanshe.

Cassirer, Ernst ([1925] 1990) Language and Myth (语言与神话, Yuyan Yu Shenhua). Trans. Yu Xiao et al. Taipei: Guiguan Chubanshe. 
Ding, Liang (2002) 'On the Characteristics of the Six Categories by Han Confucians' (论汉儒六书说之性质, Lun hanru liushu shuozhi xingzhi), in Proceedings of the 13th National Academic Symposium on Chinese Philology across the Taiwan Strait (第 十三届全国暨海峡两岸中国文字学学术研讨会论文集, Di shisanjie quanguo ji haixialiangan zhongguo wenzixue xueshu yantaohui). Taipei: Taipei Books Net Press.

Freud, Sigmund (2006) Totem and Taboo (图腾与禁忌, Tuteng Yu Jinji). Trans. Shao, Yingsheng. Taipei: Hutaomu Wenhua Chubanshe.

Huang, Rener (1955) 'On the Meaning of the Character “Yue”' (再论岳义, Zai Lun Yue Yi), Studies in Chinese Literature. 1.23.

Ji, Xusheng (2010) A New Textual Research on Origin of Chinese Characters (说文新 证, Shuowen xinzheng). Fujian: Fujian People‘s Publishing House.

Karlgren, Bernhard (1985) Chinese Language and Chinese Writing (中国语与中国 文 Zhongguoyu yu zhongguowen). Trans. Zhang, Shilu. Taipei: Taibei Wenshizhe Chubanshe.

Lakoff, George (1994) Women, Fire and Dangerous Things: What Categories Reveal about the Mind (女人，火与危险事物 Nuren huo yu weixian shiwu). Trans. Liang, Yuling et al. Taipei: Guiguan Tushu Gongsi.

Lin, Suqing (1990) 'A Study on the Artistic Calligraphy from the Spring and Autumn Period' (春秋战国美术字体研究, Chunqiu Zhanguo Meishu Ziti Yanjiu),Journal of the Institution of History and Philology 61.1.

Liu, Zhao (2011) A Study on the Structure of Ancient Chinese Characters, A Revised Version (古文字构形学[修订本], Guwenzi Gouxingxue [Xiuding Ben]). Fujian: Fujian Renmin Chubanshe.

Long, Yuchun (1994) An Introduction to Chinese Palaeography (中国文字学, Zhongguo wenzixue). Taipei: Taipei Student Book Company.

Merleau-Ponty, Maurice ([196o] 2005) Signs (符号, Fuhao). Trans. Jiang Zhihui. Beijing: Shangwu Yinshuguan.

Qiu, Xigui (1993) An Introduction to the Study on Chinese Characters (文字学概要, Wenzixue Gaiyao). Taipei: Wanjuanlou.

Saussure, Ferdinand de (2002) Saussure's Third Course of Lectures in General Linguistics. Trans. Tu Youxiang. Taizhong Longjing: Guangyang Yixue Chubanshe.

Shen, Jianshi (1935) 'On the Changes in the History of Philology of the Theory Classifying Chinese Characters According to Their Components on the Right Side, and its Applications' (右文说在训诂学上之沿革及其推阐, Youwen Shuo Zai Xunguxue Shang Zhi Yange Ji Qi TuiChan), in Institute of History and Philology (ed.), An Anthology of Academic Articles by National Academia Sinica for the 65th Birthday of Cai Yuanpei (国立中央研究院庆祝蔡元培先生六十五岁论文集, Guoli Zhongyang Yanjiuyuan Qingzhu Caiyuanpei Xiansheng Liushiwusui Lunwenji). Beijing: National Academia Sinica. 
Tang, Lan (2005) Philology on Chinese Characters (中国文字学, Zhongguo Wenzixue). Shanghai: Shanghai Guji Chubanshe.

Tu, Jiliang, ed (2006) Selected Works of Charles Sanders Peirce. Trans. Tu, Jiliang and Zhou, Zhaoping. Beijing: Shehuikexue Wenxian Chubanshe.

Wang, Ming, ed. (1960) Scriptures of the Great Peace (太平经合校, Taiping jing hejiao). Beijing: Zhonghua Book Company.

Wang, Yun (1987) Explanations on Examples from Explaining Graphs and Analysing Characters (说文释例, Shuowen Shili). Beijing: Zhonghua Shuju.

Wolfflin, Heinrich ([1915] 1987) Principles of Art History (艺术史的原则, Yishushi de Yuanze). Trans. Zeng Yayun. Taipei: Xiongshi Tushu.

Xu, Shen (1981) Notes on Explaining Graphs and Analysing Characters (说文解字注, Shuowen Jiezi Zhu). Ed. Duan, Yucai. Shanghai: Shanghai Classics Publishing House. Xunzi (1988) Collected Commentary of Xunzi (荀子集解, Xunzi Jijie). Ed. Wang, Xianqian. Taipei: Yiwen Yinshuju.

Xu, Zhongshu (1981) Forms of Ancient Chinese Writing (汉语古文字字形表, Hanyu Guwenzi Zixingbiao). Chengdu:Sichuan People's Publishing House.

Yan, Zhitui (199o) Collected Commentary on the Family Instructions from Yan Zhitui (严 氏家训集解, Yanshi Jiaxun Jijie). Ed. Wang, Liqi. Taipei: Mingwen Shuju.

Zheng, Xuan, and Jia, Gongyan (1989) Notes and Commentary on Rites of the Zhou Dynasty (周礼注疏, Zhouli Zhushu). Ed. Ruan, Yuan. Taipei: Dahua Chubanshe.

Zhuang, Zhou (1991) Collected Notes on Zhuangzi (庄子集释, Zhuangzi Jishi). Ed. Qingpan Guo. Taipei: Qunyu Tang. 